\title{
UNA SEPULTURA ARGARICA DE EL VERDOLAY (MURCIA)
}

ANA M. ${ }^{a}$ MUÑOZ Amilibia

En este homenaje que tributamos al Profesor Don Gratiniano Nieto Gallo, he querido dar noticia de una tumba argárica en primer lugar por su procedencia, el poblado de Santa Catalina del Monte, a cuya necrópolis, el Cabecico del Tesoro, dedicó tantos años de su vida, y también porque fue precisamente el Profesor Nieto el que publicó por primera vez la noticia del descubrimiento de una necrópolis argárica en la ladera del monte de Santa Catalina, junto a la ermita de San Antonio El Pobre (1). El descubrimiento se hizo con motivo de las primeras campañas de excavación en la necrópolis del Cabecico del Tesoro en El Verdolay, dirigidas por Don Cayetano de Mergelina y de las prospecciones que se hicieron en su entorno. En la citada publicación se ilustra el momento de la aparición de las dos grandes jarras de enterramiento y las urnas ya reconstruidas. En segundo lugar por el interés que puede tener siempre el conjunto cerrado del ajuar de una sepultura argári$\mathrm{ca}$, en este caso en cista.

El yacimiento situado en el monte de Santa Catalina ofrece restos de poblamiento por lo menos desde el Eneolítico con vaso campaniforme (2) hasta bien entrada la romanización. Su conocimiento se debe a trabajos de prospección, como el ya mencionado, o a hallazgos mas o menos casuales. Lamentablemente una gran parte del poblado, coronado por el castillo árabe llamado de La Luz, fue cedido por el Ayuntamiento a particulares. Hacia 1956 se construyó una carretera de acceso desde el Convento de Santa Catalina del Monte a la urbanización proyectada en la ladera norte del cerro, cortando los depósitos estratificados que dejaban ver claramente la larga secuencia del poblado: urnas y cistas argáricas que fueron destrozadas, cerámica ibérica y romana (3). La edificación posterior de viviendas resi-

(1) Nieto Gallo, G: «Noticia de las excavaciones realizadas en la necrópolis hispánica del Cabecico del Tesoro, Verdolay (Murcia)w, Bol. del Seminario de Arte y Arq. de la Universidad de Valladolid, Tomo VI (1940), pág. 138, láms. IV y V.

(2) Fernández Avilés, A.: «Materiales de Arqueología murciana. Dos fragmentos de cerámica incisa procedentes de Murcian, Boletín del Museo de Bellas Artes, Murcia, 1935.

(3) Jorge Aragoneses, M.: "La badila ritual ibérica de La Luz (Murcia) y la topografía arqueológica de aquella zona según los últimos descubrimientos», Anales de la Universidad de Murcia XXVI (2), 1968, págs. 18-21, figs. 27 y 28. 
denciales no hicieron mas que confirmar el hecho y al mismo tiempo destruir una parte del yacimiento. Concretamente, en 1966, con motivo de la construcción del chalet de Don Antonio González Conte, se pudo comprobar, en un depósito de 2,50 a 3,50 metros, los restos del poblado ibérico y por encima los de un asentamiento romano interpretado como una villa (4).

A pesar de las gestiones del Departamento de Arqueología, desde el año 1976, para impedir nuevas construcciones, una Orden Ministerial, de fecha 27-12-1977, calificaba la ladera norte del poblado como "ciudad jardín" y "espacios forestales privados", hecho que confirmó, ya de forma legal, el desarrollo de la urbanización de tipo residencial, en un paraje verdaderamente privilegiado, pero que afectó y sigue afectando gravemente con nuevas construcciones, al yacimiento arqueológico.

En una de las numerosas visitas realizadas al lugar, tomamos contacto con el propietario de una de las primeras residencias construidas por los años sesenta, en la zona próxima a la ermita de San Antonio el pobre, Don Angel de La Fuente, quien nos manifestó que, al construir el frontón de su casa había aparecido una cista de lajas de piedra, de la que conservaba el ajuar, que amablemente nos entregó para su estudio, y del que ahora nos ocupamos (fig. 1 y 2,1).

El ajuar estaba compuesto por cuatro vasijas cerámicas, un hacha y un puñal que a continuación se describen (fig. 2,2 y 3).

1. Hacha de cobre o bronce de forma trapezoidal con el filo ensanchado y curvo; en los lados presenta un ligero reborde que sólo sobresale un milímetro; el talón, que aparece roto, sólo alcanza cuatro milímetros de grosor en la parte más alta conservada. Mide $172 \mathrm{~mm}$. de longitud máxima, $45 \mathrm{~mm}$. de anchura en el talón y $98 \mathrm{~mm}$. de anchura máxima en el filo. Es de sección muy plana, extremadamente delgada, sobre todo en el filo, con un grosor entre 2 y $3 \mathrm{~mm}$. Por su tipología se encuadraría en el grupo C, argárico, de Siret (5), en el algo más preciso III de Cuadrado (6) y en el I de Blance (7), pero evidentemente queda fuera de las tipologías más comunes establecidas para las hachas argáricas. También queda fuera de los dos tipos dados por Lull pues el índice de ángulo da 0,274, el de exvasamiento del filo 0,489 y sobre todo un área de $132,86 \mathrm{~cm}^{2}$ (8), lo que la aleja de los tipos característicos de este autor. Aunque todavía no disponemos de los resultados del análisis del metal, si se pueden observar ciertos detalles técnicos de interés. La esfoliación que presenta en capas en la parte rota del talón, su sección plana y extremadamente fina, así como los rebordes laterales, parecen indicar que ha sido trabajada por batido, aunque pudiera haberse moldeado previamente. Por otro lado, las características citadas hacen pensar que se tratara de un arma mas que de un útil, cumpliendo la misma función que una alabarda (9).

(4) Jorge Aragoneses, M.: 1968, cit.

(5) Siret, L.: "Questions de Chronologie et d'Etnographie iberiques», Paris, 1913, pág. 334.

(6) Cuadrado Díaz, E.: "Utiles y armas de El Algar. Ensayo de tipologíaw. I CNA y V C Arq. del Sudeste (Almería 1949), Cartagena, 1950, pág. 120.

(7) Blance, B.: Die Anfange der Metallurgie auf der Iberischen Halbinsel, Berlin, 1971, págs. 126-127 „lám. 23.

(8) Lull, V.: La cultura de El Argar. Akal, Madrid, 1983, pág. 180, gráficos 85-87.

(9) Siret, L.: 1913, cit. pág. 343. 


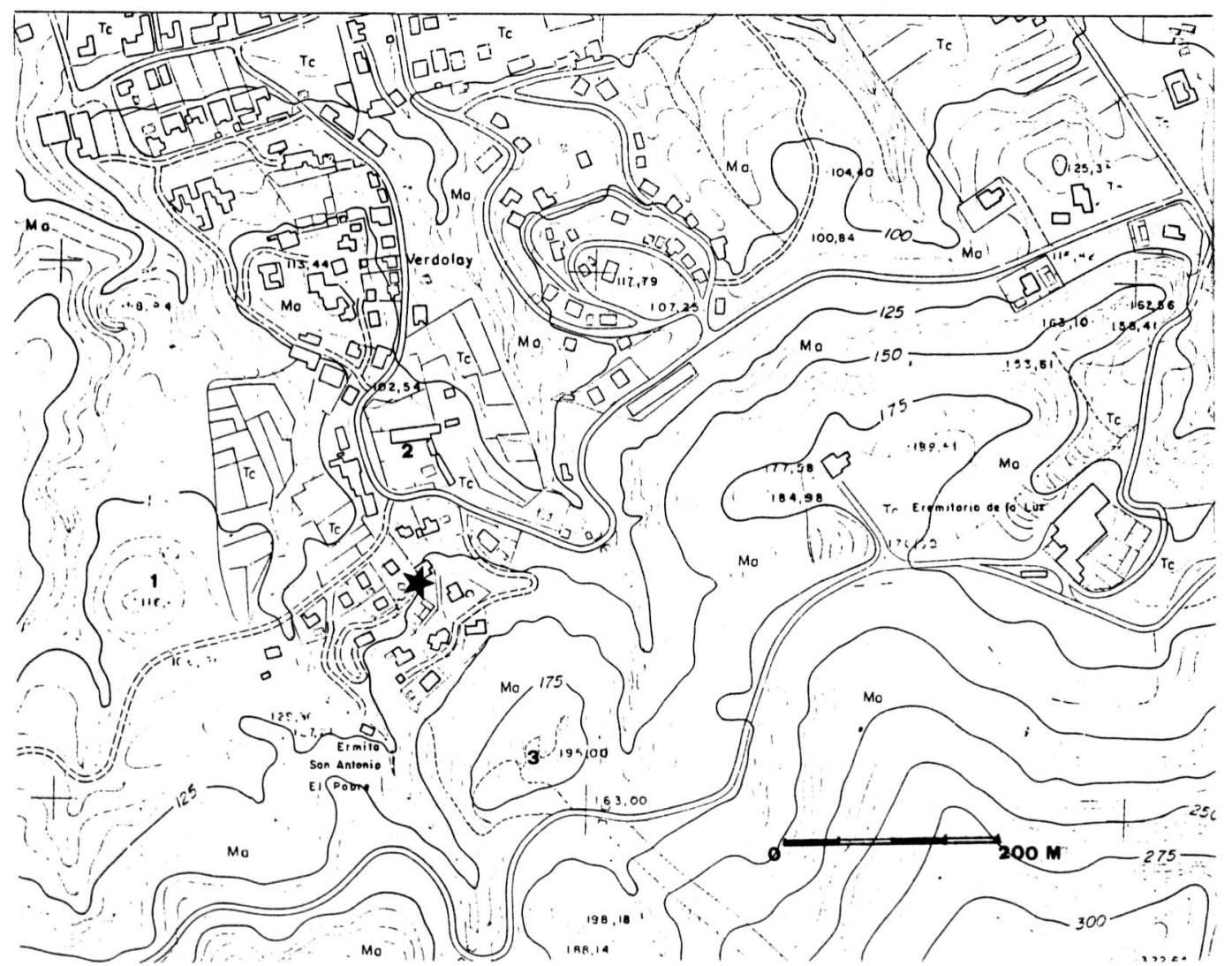

FIG. 1.-1. Necrópolis del Cabecico del Tesoro; 2. Convento de Santa Catalina del Monte; 3. Castillo de Santa Catalina o de La Luz. La estrella indica el lugar de hallazgo de la sepultura. (Plano aerofotogramétrico escala 1:5000, equidistancia de curvas, $5 \mathrm{~m}$. Hoja 943-2-4. Ayuntamiento de Murcia). 

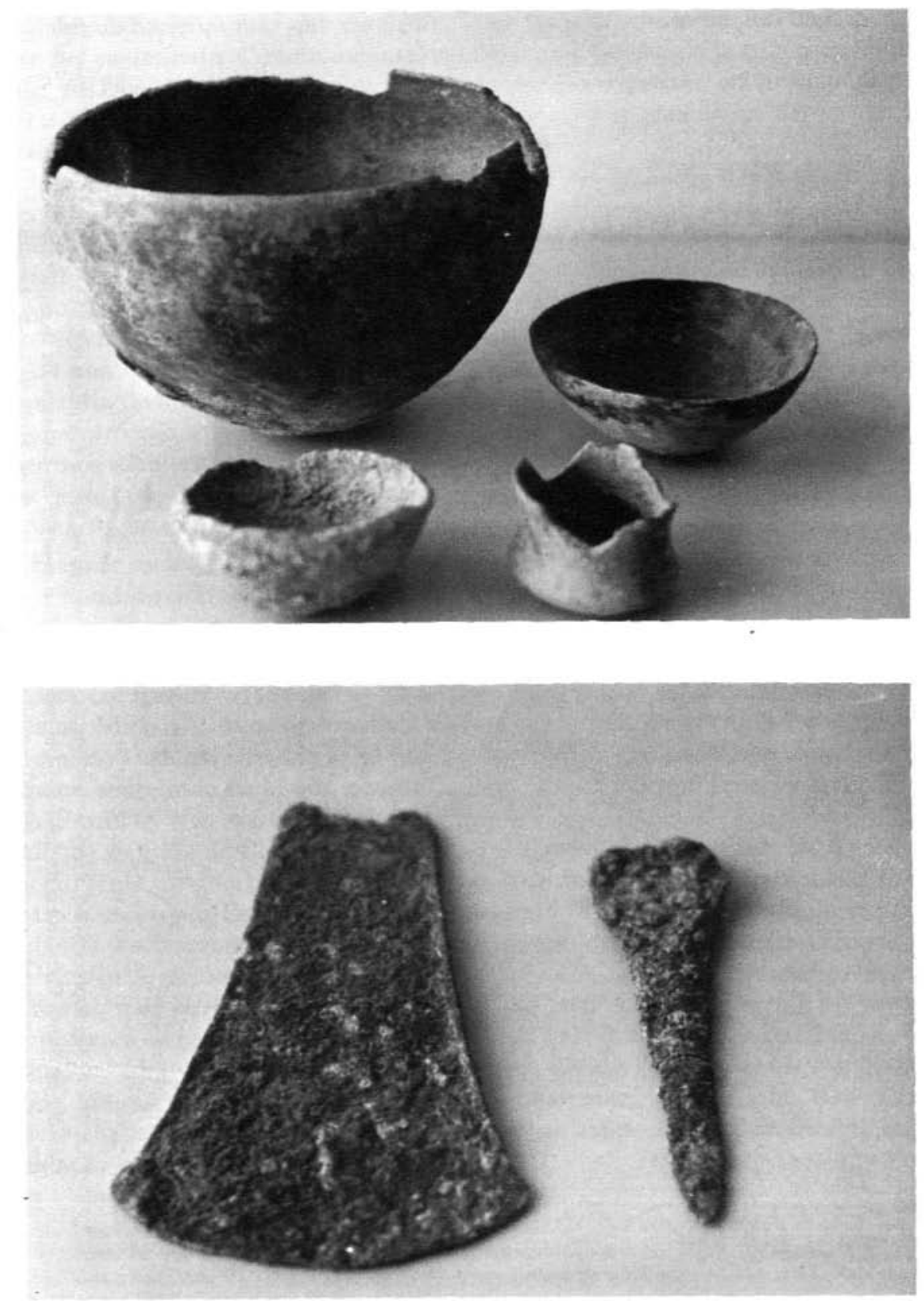

FIG. 2.-1. Frontón de la casa de Don Angel de La Fuente, en cuya construcción apareció la sepultura; arriba chalet del Sr. Pérez Bas. 2. Ajuar de la sepultura. 

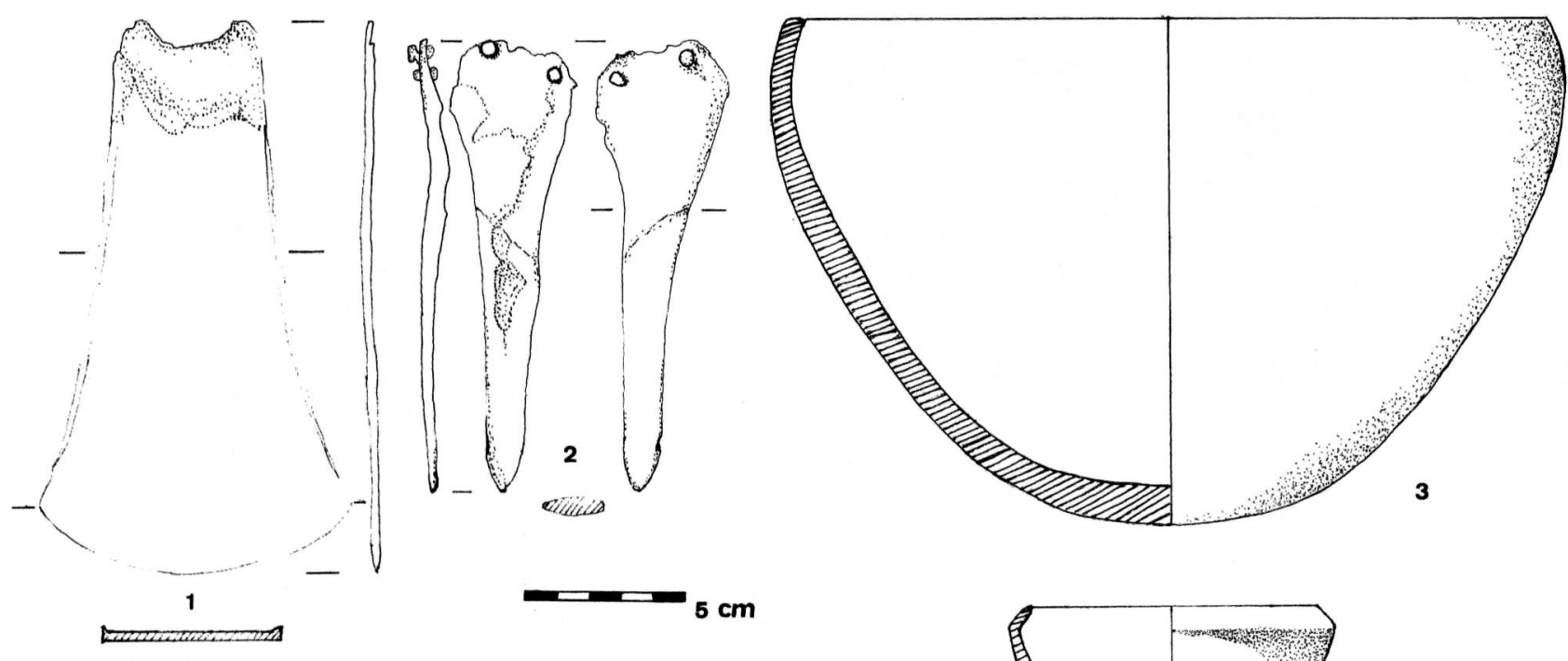

$\vec{w}$

FIG. 3 ,
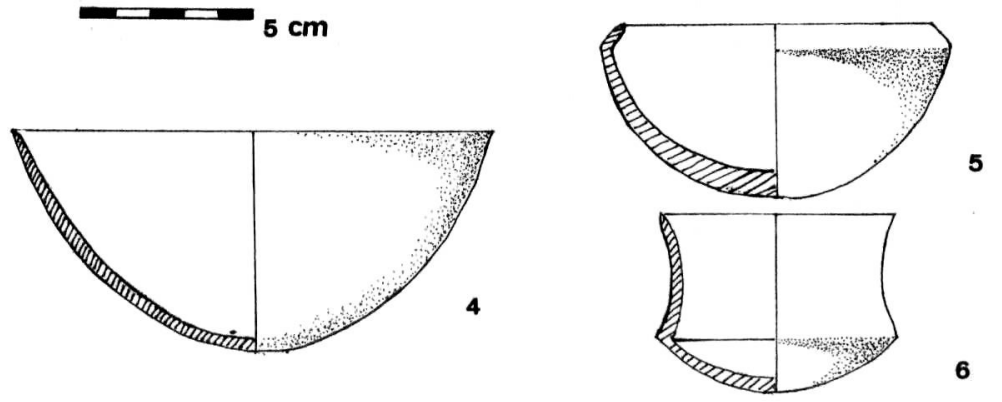

\author{
(
}


2. Puñalito de cobre o bronce de base redondeada con dos remaches, que se va estrechando hasta ofrecer una punta aguzada. Mide $141 \mathrm{~mm}$ de longitud y 40 $\mathrm{mm}$ de anchura máxima. Su grosor oscila entre $1 \mathrm{~mm}$. en la punta y $6 \mathrm{~mm}$. en el centro donde presenta la sección ovoidal. Está roto y pegado por su parte media y en uno de sus lados presenta la esfoliación de la capa superior del metal, estando ligeramente curvado y roto en el borde de la base de enmangue. Aunque no se ha tratado en Laboratorio parece tener restos de tejido junto a uno de los remaches. Por su tipología podría encuadrarse dentro del tipo II de Blance (10).

3. Cazuela cerámica muy honda con borde reentrante y fondo de tendencia parabólica, Forma 2 de Siret. De pasta marrón rojiza, muy esfoliable, con grueso desgrasante y superficies interna y externa con engobe negruzco espatulado. Mide $157 \mathrm{~mm}$. de altura, $235 \mathrm{~mm}$. de diámetro en la boca y $247 \mathrm{~mm}$. de diámetro máximo a $22 \mathrm{~mm}$. del borde. Su grosor oscila de $5 \mathrm{~mm}$. en el borde a $13 \mathrm{en}$ el fondo. Le faltan algunos fragmentos del borde.

4. Cuenco parabólico de boca ensanchada, Forma 1 bis de Siret. Pasta muy fina roja y engobe interior y exterior bruñido de color gris oscuro, casi negro. Mide $64 \mathrm{~mm}$. de altura, $140 \mathrm{~mm}$. de diámetro en la boca, y las paredes, muy finas, miden $1 \mathrm{~mm}$. en el borde y $4 \mathrm{~mm}$. en el fondo. Está casi completa.

5. Cuenco de borde reentrante de la Forma 2 de Siret, de pasta gris verdosa con desgrasante grueso de cuarzo muy patente sobre todo en el borde, muy deteriorado, donde ha perdido el engobe gris espatulado, que cubre su superficie interna y externa. Altura $50 \mathrm{~mm}$., diámetro en la boca $90 \mathrm{~mm}$. y máximo $120 \mathrm{~mm}$. en la carena en que quiebra el borde. El grosor oscila entre los $2 \mathrm{~mm}$. del borde y los $8 \mathrm{~mm}$. del fondo.

6. Vasito carenado de la Forma 5 de Siret. Pasta beige ennegrecida muy fina y compacta con restos de bruñido negro en la superficie interior. Le faltan unos dos tercios del borde. Altura $52 \mathrm{~mm}$., diámetro en la boca $68 \mathrm{~mm}$. y máximo en la línea de $71 \mathrm{~mm}$. La carena, baja, está a $15 \mathrm{~mm}$. del fondo y $37 \mathrm{~mm}$. del borde.

Como se ha mencionado al principio, el ajuar procedía de una tumba de inhumación en cista y, aunque el Sr. de la Fuente conservó durante un tiempo el cráneo, ya no lo tenía cuando nos hizo entrega de los materiales. Hemos visto que por la misma zona aparecieron enterramientos argáricos en urnas, pero no se menciona que estas tuvieran ajuar (11), por lo que no se pueden hacer deducciones de tipo cronológico derivadas de la estructura de la sepultura. En cuanto al ajuar que estudiamos, el primer elemento a destacar es la presencia de dos vasijas de la Forma 2 , forma que parece ser una de las mejor representadas en los enterramientos en urna, un $14 \%$, frente a un $4 \%$ en las cistas (12). La forma 1 en cambio, sería poco significativa, con un $13 \%$ en ambos tipos de enterramiento. En cuanto a la forma 5 , que en nuestro caso presenta la carena baja, es más frecuente en cistas, un $59 \%$, que en urnas, un $28 \%$ (13).

(10) Blance, B.: 1971, cit. pág. 124.

(I1) . Nieto, G.: 1940, cit. pág. 138.

(12) Lull, V.: 1983, cit. págs. 148, 151 y 154.

(13) Lull: 1983 , cit. pág. 154. 

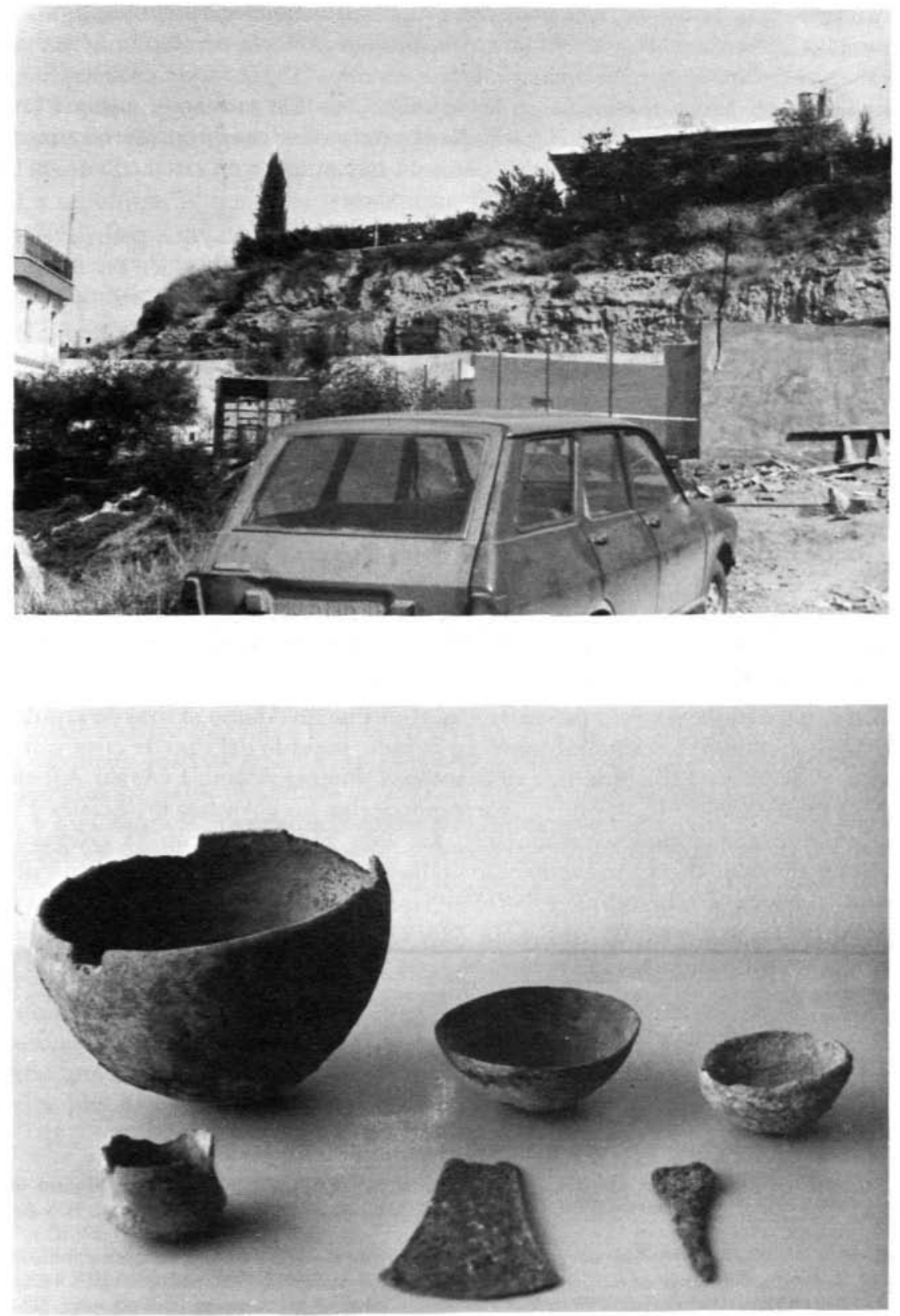

FIG. 4.-Piezas de cerámica y metal que componían el ajuar. 
La presencia del hacha, que para Siret representaría un momento antiguo, anterior a las alabardas (14), para Blance en cambio supondría una fase más reciente, Argar B, relacionada con los enterramientos en urna (15), aunque en nuestro caso la tipología del hacha no encaja en las establecidas. Sin embargo, aunque en El Argar y en la Bastida de Totana el hacha parece estar relacionada con enterramientos en urna, no ocurre lo mismo en otros casos en que aparece en cistas, como en Callosa de Segura, El Oficio (16) o en Fuente Alamo.

Finalmente, el puñal con dos remaches y base redondeada, que podría relacionarse con el tipo II de Blance (17) o el A de Cuadrado (18), parece ser frecuente en las cistas y por tanto del Argar A de la citada autora.

De todo ello podrían deducirse las siguientes conclusiones. Se trata de una cista con un puñal y dos vasos cerámicos - Formas 1 y 5 - habituales en este tipo de enterramiento, mientras que las vasijas de la Forma 2 y el hacha parecen ser más frecuentes en los enterramientos en urna. De todas formas, la tipología del hacha se sale de los eşquemas normales en tipos argáricos, de talón más estrecho y sección gruesa. Más difícil es inferir conclusiones de tipo cronológico en el estado actual de la cuestión en lo referente al Bronce argárico, pero voy a intentarlo de acuerdo con los datos que nos ofrecen las excavaciones de Fuente Alamo, en donde las sepulturas han podido estudiarse en función de su posición estratigráfica y del propio análisis que ofrece el estudio de los conjuntos cerrados de sus ajuares (19), siempre con la prudencia de no generalizar los resultados aportados por un yacimiento concreto a toda el área argárica.

En primer lugar, parece confirmarse que en Fuente Alamo el tipo de sepultura más antiguo es el de covacha excavada en la roca, seguido del tipo de cista y, finalmente, el de pithos (20). A la fase más antigua, Fuente Alamo I (Argar A), entre el 2000/1900-1800/1750 antes de C., corresponderían las covachas artificiales y una cista grande con dromos (tumba 69). La fase Fuente Alamo II (Argar A), 1800/1750-1650/1600 a. C., de momento no ha documentado sepulturas. En Fuente Alamo III (Argar B), 1650/1600-1500/1450, las sepulturas son de cista, mientras que en la Fase Fuente Alamo IV (Argar B), 1500/1450-1400/1350, predominan los enterramientos en urna, aunque hay dos cistas de tipo pequeño. Todavía no se ha publicado la totalidad de los ajuares para poder establecer posibles comparaciones con

(14) Siret, L.: 1913, cit. pág. 343.

(15) Blance: 1971, cit. pág. 131.

(16) Lull: 1983, cit. págs. 189-190.

(17) Blance: 1971, cit. págs. 124-125.

(18) Cuadrado Díaz, E.: 1950, cit. pág. 121, fig. 12.

(19) Arteaga, O. y Schubart, H.: «Fuente Alamo. Excavaciones de 1977», N. Arq. H., 9, Madrid, 1980, págs. 245-289. Idem, "Campaña de 1979n, N. Arq. H. 11, Madrid, 1981, págs. 8-33. Schubart, H. y Arteaga, O.: "Excavaciones en Fuente Alamo», Revista de Arqueología, 1983; I, n. ${ }^{\circ} 24$, págs. 17-27; II, n. ${ }^{\circ} 25$, págs. 54-63; III, n. ${ }^{\circ} 26$. Idem, «Fundamentos arqueológicos para el estudio socio-económico y cultural del área de El Argar», Homenaje a Luis Siret (Cuevas del Almanzona, 1984), 1986, págs. 289-307. Schubart, H.: "Consideraciones sobre el Bronce del Sureste Peninsular». Historia de Cartagena, Tomo II, págs. 219-250, Edic. Mediterráneo, Murcia, 1986.

(20) Schubart, H.: 1986, cit. págs. 236-240. 
el que estudiamos, pero voy a referirme a algunas sepulturas de las que se han dado más noticias. De la fase FA III, la tumba 52, en cista, excavada en 1977, que estratigráficamente puede corresponder al Argar A avanzado, y la tumba 65, excavada en 1979, que apareció en el corte 2. De la fase FA IV, la tumba 68, cista pequeña, excavada en 1979, que es una tumba femenina como las dos anteriores, y con un rico ajuar (21).

La sepultura 52 tenía un ajuar compuesto por un gran vaso bicónico, Forma 6 de Siret, frecuente en la fase Argar A, y un vasito de la forma 5 con la carena baja muy detacada, frecuente en el Argar B, de lo que sus excavadores deducen que la sepultura correspondería a una fase antigua del Argar B, de acuerdo también con los objetos metálicos del ajuar: un puñalito de dos remaches que conserva restos de la empuñadura, un punzón y dos anillos de plata en espiral (22).

La sepultura 65 , también de cista, contenía como la anterior un vaso de la forma 6 y otro vasito de la forma 5 , semejante al de la sepultura 52, además de tres anillos, un punzón y un puñal con dos remaches, que no se reproducen (23).

La sepultura 68, en cista, apareció en el corte 7 y al parecer podría corresponder al Argar B. Contenía, además del esqueleto de una mujer joven, dos vasijas de forma 4, un vasito carenado de la forma 5 "tardío", una copa de pie esbelto, un anillo y un brazalete de plata, un puñal de tres remaches y un hacha plana (24).

Como vemos, se trata de tres sepulturas de cista. La 52, aunque se sitúa en la fase FA III, estratigráficamente podría pertenecer al Argar A avanzado, lo que estaría de acuerdo con el vaso de la forma 6 como elemento antiguo, lo mismo que la sepultura 65 que se sitúa en la fase FA III. Su adscripción a la fase IV en el cuadro de análisis metálicos podría ser una errata (25). De todas formas, la sepultura 68 queda adscrita a FA IV, en pleno Argar B o B2. De todo ello se puede inferir que pueden darse sepulturas en cista, aunque sean pequeñas en el Argar pleno o B, y que algunos elementos considerados antiguos, como la forma 6 cerámica, debieron de pervivir como la propia tradición de enterramiento en cista. No hay que descartar el que el enterramiento en cista puede depender de la posibilidad de obtener las losas necesarias para su construcción, siempre más costosa que la covacha o sobre todo la jarra cerámica, y no necesariamente la sustitución del tipo se pudo hacer en todos los poblados al mismo tiempo.

Por ahora no se puede establecer un paralelismo concreto entre el ajuar de nuestra sepultura y los publicados de Fuente Alamo, pero a modo de hipótesis podría admitirse que se tratara de una cista perteneciente a un momento avanzado del Argar, Bronce pleno, y encuadrarse cronológicamente entre el 1600 y el 1400 a. de C.

(21) Schubart y Arteaga: 1986, cit. págs. 292 y 298-299.

(22) Arteaga y Schubart: 1980 , cit. págs. 261 y fig. 9.

(23) Arteaga y Schubart: 1981, cit. pág. 15.

(24) Arteaga y Schubart: 1981, cit. fig. 5 y lám. 10.

(25) Schubart y Arteaga: 1986, cit. págs. 298 y 307. 